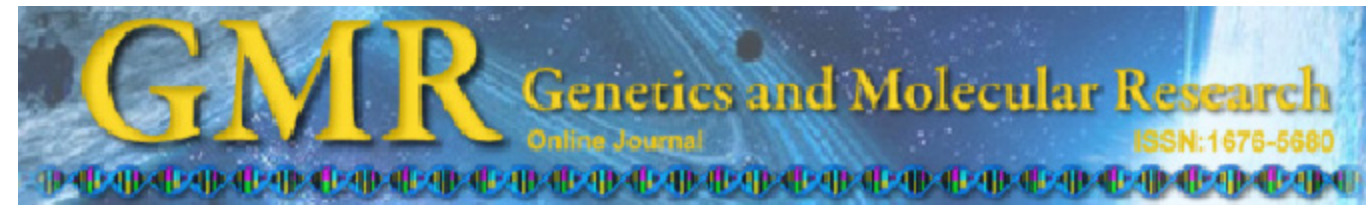

$\underline{\text { Case Report }}$

\title{
Muscle pain, fever, cough, and progressive dyspnea in a woman with eosinophilic pneumonia
}

\author{
Z. Xu, Y. Fan, G.S. Wang and G.M. Wu \\ Respiratory Disease Department, Xinqiao Hospital, \\ Third Military Medical University, Chongqing, China \\ Corresponding author: G.M. Wu \\ E-mail: guomingwucn@yeah.net
}

Genet. Mol. Res. 14 (2): 4189-4194 (2015)

Received June 27, 2014

Accepted December 12, 2014

Published April 28, 2015

DOI http://dx.doi.org/10.4238/2015.April.28.1

\begin{abstract}
The diagnosis of eosinophilic pneumonia (EP) is rare and challenging. This condition is frequently misdiagnosed as pulmonary tuberculosis, lymphoma, schistosomiasis, Wegener's granuloma, severe acute respiratory syndrome, or severe communityacquired pneumonia. Herein, we report a case in which computed tomography (CT)-guided percutaneous lung biopsy was used to diagnose EP without alveolar eosinophilia or peripheral eosinophilia. A roundworm identified in the patient's stool confirmed the precise diagnosis to be parasitic EP. This is, to our knowledge, the first reported case of EP confirmed by CT-guided percutaneous lung biopsy. CT-guided percutaneous lung biopsy may represent a new tool for the diagnosis of EP in patients without typical alveolar eosinophilia or peripheral eosinophilia.
\end{abstract}

Key words: Parasitic eosinophilic pneumonia; Percutaneous lung biopsy; Diagnosis 


\section{INTRODUCTION}

Parasitic eosinophilic pneumonia (EP) without alveolar eosinophilia or peripheral eosinophilia remains a rare condition and is challenging to diagnose. The condition is frequently misdiagnosed as pulmonary tuberculosis, lymphoma, schistosomiasis, Wegener's granuloma, severe acute respiratory syndrome, or severe community-acquired pneumonia. Herein, we present a case of a 58-year-old patient diagnosed with parasitic EP and a review of the literature on this topic.

\section{CASE REPORT}

A 58-year-old woman was admitted to the Respiratory Disease Department at our hospital because of myalgia and fever (maximum temperature: $39^{\circ} \mathrm{C}$ ) for 20 days and cough with progressive dyspnea for 10 days. Her pneumonia had been diagnosed at a local community hospital and then treated with azithromycin and piperacillin for 3 days, but her symptoms did not resolve. She had lost about $5 \mathrm{~kg}$. There was no relevant occupational or travel history. She did not take any medications on a regular basis. On admission, a physical examination showed a body temperature of $39.3^{\circ} \mathrm{C}$. Moist crackles were noted in the right lower lung on auscultation. No abnormalities were found in the heart or abdomen.

The initial investigation showed an elevated erythrocyte sedimentation rate (ESR $93 \mathrm{~mm} / \mathrm{h}$ ). The results of routine blood test results were normal. Arterial blood gas tension showed type I respiratory failure $\left(\mathrm{pH} 7.375 ; \mathrm{pCO}_{2} 45.0 \mathrm{mmHg}\right.$; $\mathrm{pO}_{2} 57 \mathrm{mmHg}$; and $\mathrm{HCO}_{3}^{-}$ $26.5 \mathrm{mM}$ ). The results of pulmonary function tests showed: 1 . moderate to severe mixed ventilatory dysfunction; 2. compromised small airway function; 3. reduced total lung capacity (TLC) and vital capacity (VC), increased residual volume (RV)/TLC\%, and normal RV and functional residual capacity (FRC); and 4. increased airway resistance. Anti-nuclear antibody, anti-neutrophil cytoplasmic antibody, anti-glomerular basement membrane antibody, and PPD tests were all negative. The levels of cancer markers were normal. Acid-fast bacilli examination of the sputum was negative (7 times). Blood bacterial culture and bacterial culture of the sputum were negative. Examination of the stool showed no eggs. Bronchoscopy showed no evident abnormalities. Bronchoalveolar lavage (BAL) fluid was negative for acid-fast bacilli. The white blood cells identified in BAL fluid were: $55.1 \%$ monocytes/macrophages; $7.2 \%$ lymphocytes; $0.9 \%$ eosinophils; and $36.8 \%$ neutrophils.

On admission, computed tomography (CT) of the chest and air bronchography (Figure 1A) revealed massive exudates and consolidation in the right lower lung. At 6 (Figure 1B) and 14 days (Figure $1 \mathrm{C}$ and D) after admission, chest $\mathrm{CT}$ results indicated enlargement of the lesions in her right lower lung, and new exudative lesions and consolidation were present in her left lower lung and right upper lung. We therefore speculated that this was not bacterial pneumonia but rather pneumonia due to some other cause(s) associated with infiltrative lesions, such as caseous tuberculous pneumonia, Goodpasture's syndrome, allergic pneumonia, or EP.

To confirm the diagnosis, CT-guided percutaneous lung biopsy and pathological examination were performed. Pathology results revealed increased alveolar septa and eosinophil infiltration into the submucosa of the bronchioles and interstitium (Figure 2). In addition, a roundworm was identified in the patient's stool 16 days after her admission. These findings confirmed the diagnosis of parasitic EP. Oral albendazole $(0.4 \mathrm{~g})$ was administered once as 
anti-parasitic treatment and oral methylprednisolone $(40 \mathrm{mg})$ was given once daily as antiinflammation therapy. The patient's cough and dyspnea improved subsequently. One month later, the exudative lesions had disappeared completely (Figure 3 ).

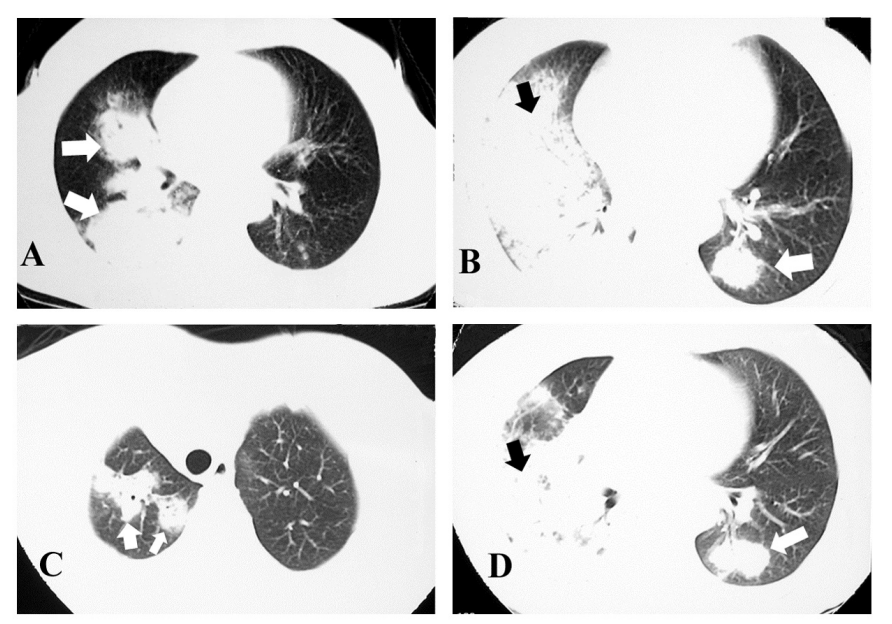

Figure 1. A. On admission, chest $\mathrm{CT}$ revealed massive exudates and consolidation in the right lower lung as well as air bronchograms (arrowed). B. At 6 days after her admission, chest CT indicated enlargement of the lesions in her right lower lung, and new exudative lesions in her left lower lung (arrowed). C. D. At 14 days after her admission, new exudative lesions were present in right upper lung and the lesions in her left lower lung became larger (arrowed).
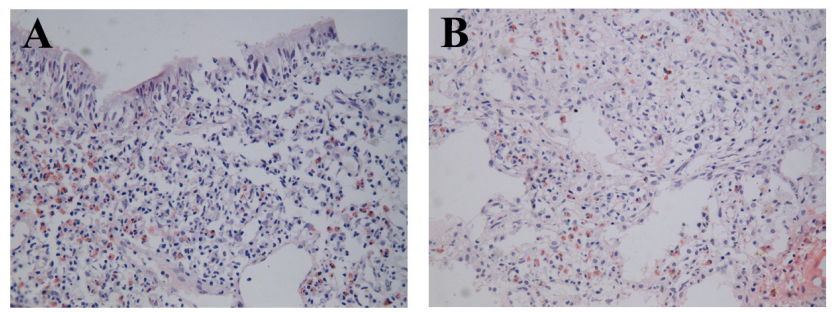

Figure 2. A. Numerous eosinophils had infiltrated into the submucosa of bronchioles, H\&E 200X; and B. detail of the septal alveolar thickening caused by eosinophils infiltration, H\&E 200X.

A

B
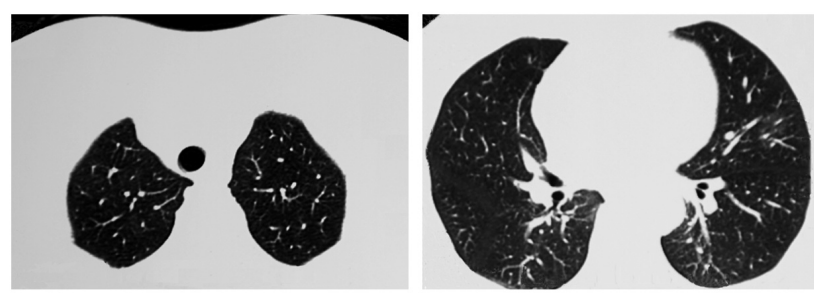

Figure 3. Infiltration shadows in right upper lung (A) and bilateral lower lung (B) had completely disappeared. 


\section{DISCUSSION}

EP comprises a heterogeneous group of lung diseases characterized by the accumulation of eosinophils in the pulmonary interstitium and airspaces, with or without increased levels of eosinophils in peripheral blood (Campos and Pereira, 2009). These lung diseases can be classified as follows: EP of unknown cause [simple pulmonary eosinophilia (SPE), Loffler's syndrome], idiopathic chronic and acute EP, Churg-Strauss syndrome (CSS), or the [hypereosinophilic syndromes (HES)]; EP of determined cause such as a parasitic infection, drug reaction, allergic bronchopulmonary aspergillosis (ABPA), and bronchocentric granulomatosis (BG) (Cottin and Cordier, 2005).

Although infiltration of the lung by eosinophils is an important marker for the diagnosis of EP, the inflammatory infiltrate is also composed of macrophages, lymphocytes, and neutrophils. All of these cells induce lung injury and impair gas exchange. Inflammation involves eosinophil-released mediators as well as chemokines, complement proteins, and other chemoattractants (Samitas et al., 2011). The host's immune response to the antigenic constituents of circulating microfilariae trapped in the lung vasculature is another notable aspect of parasitic EP.

The main symptoms of EP are cough, dyspnea that may be associated with fever, weight loss, general malaise, and anorexia. Among these symptoms, cough and dyspnea are the most frequent (Al-Jahdali et al., 2012). Acute hypoxemic respiratory failure and airway hyperresponsiveness can manifest in combination as an asthma-like syndrome (Vijayan, 2007). This patient presented with fever, cough with progressive dyspnea and weight loss, as well as muscle pain. According to the literature, muscle pain is a rare symptom of EP, which has not yet been reported.

Therapy for EP consists of long-term treatment with systemic corticosteroids, which usually achieves a dramatic response. However, relapses are frequent when tapering or discontinuing treatment. Therefore, long-term systemic corticosteroid therapy should be performed in combination with more targeted treatment approaches. For instance, parasitic EP is typically treated with anthelminthics; the effects of additional corticosteroid treatment could be beneficial (Boland et al., 2011; Ray et al., 2012). In this case, oral albendazole (0.4 g) was administered once for anti-parasitic treatment; oral methylprednisolone $(40 \mathrm{mg})$ was given once daily as anti-inflammation therapy. The patient's cough and dyspnea improved subsequently. One month later, the exudative lesions had disappeared completely.

Pulmonary infiltrates in EP can be identified using chest radiography or chest highresolution CT (HRCT) scans. Pulmonary infiltrates typically present as focal areas with a ground-glass appearance and irregularly shaped nodular consolidations on air bronchograms (Martins et al., 2008; Kalogeropoulos et al., 2011). The radiological findings associated with EP differ based on the condition's etiology. Several studies have examined the CT findings in acute EP (AEP). The CT findings in AEP patients consisted mainly of bilateral patchy areas of ground-glass attenuation, interlobular septal thickening, thickening of the bronchovascular bundles, and the presence of pleural effusion without cardiomegaly (Cheon et al., 1996). Unlike AEP, bilateral air-space consolidation with peripheral predominance was the most frequent CT finding in CEP, followed by linear and reticular opacities and ground-glass opacities (Mayo et al., 1989; Arakawa et al., 2001). HRCT manifestations of drug-induced EPs resemble those of chronic EP, bilateral air-space consolidation, peripherally distributed ground- 
glass opacities, nodules, and irregular lines (Souza et al., 2006). In the case presented here, the pulmonary infiltrates sometimes displayed multiple migratory shadows. Furthermore, in some cases of allergic bronchopulmonary aspergillosis (ABPA), Churg-Strauss syndrome (CSS), and parasitic infection, pleural effusion and/or cavitation can occur.

$\mathrm{PE}$ is frequently misdiagnosed as pulmonary tuberculosis, lymphoma, schistosomiasis, Wegener's granuloma, severe acute respiratory syndrome, or severe community-acquired pneumonia (Katz and Shoenfeld, 2008). The diagnosis of EP requires both typical radiological features and alveolar eosinophilia with or without peripheral eosinophilia. Increased eosinophil counts in the bronchoalveolar lavage fluid (BALF) confirm a diagnosis of EP (Fernández Pérez et al., 2011). However, in some difficult cases, such as our case, the alveolar eosinophils and peripheral eosinophils are found only in pulmonary infiltrates. A lung biopsy will be necessary to establish the diagnosis of EP in these cases (Abe et al., 2003). The typical histopathological findings include alveolar space infiltration by eosinophils and the septal alveolar thickening caused by eosinophil and mononuclear cell infiltration, as well as macrophages containing Charcot-Leyden crystals. In our case, the results of the lung biopsy confirmed the histopathological characteristics of EP.

\section{CONCLUSION}

In summary, we present a case of EP secondary to Ascaris lumbricoides infection. The patient's clinical course, radiographic, and laboratory findings strongly supported a diagnosis of severe community-acquired pneumonia. However, emerging pulmonary infiltrations appeared in the right upper lobe and left lower lobe after antimicrobial therapy for 5 days. Although the migratory pulmonary infiltrations confirmed the suspected diagnosis of EP, the alveolar eosinophilia and peripheral eosinophilia necessary for definitive confirmation were absent. EP without alveolar eosinophilia or peripheral eosinophilia remains a rare but challenging condition. Clinicians faced with these difficult cases should keep several possibilitiesincluding EP-in mind as differentials in the diagnosis of lung disease.

\section{ACKNOWLEDGMENTS}

The authors thank Professor Yi Xianghua for his pathology figure.

\section{REFERENCES}

Abe K, Yanagi S, Imadsu Y, Sano A, et al. (2003). Acute eosinophilic pneumonia with fine nodular shadows. Intern. Med. 42: 88-91.

Al-Jahdali H, Waness A, Al-Jawder S, Baharoon SA, et al. (2012). Eosinophilic pneumonia: experience at two tertiary care referral hospitals in Saudi Arabia. Ann. Saudi Med. 32: 32-36.

Arakawa H, Kurihara Y, Niimi H, Nakajima Y, et al. (2001). Bronchiolitis obliterans with organizing pneumonia versus chronic eosinophilic pneumonia: high-resolution CT findings in 81 patients. AJR Am. J. Roentgenol. 176: 1053-1058.

Boland JM, Vaszar LT, Jones JL, Mathison BA, et al. (2011). Pleuropulmonary infection by Paragonimus westermani in the United States: a rare cause of Eosinophilic pneumonia after ingestion of live crabs. Am. J. Surg. Pathol. 35: 707-713.

Campos LE and Pereira LF (2009). Pulmonary eosinophilia. J. Bras. Pneumol. 35: 561-573.

Cheon JE, Lee KS, Jung GS, Chung MH, et al. (1996). Acute eosinophilic pneumonia: radiographic and CT findings in six patients. AJR Am. J. Roentgenol. 167: 1195-1199. 
Cottin V and Cordier JF (2005). Eosinophilic pneumonias. Allergy 60: 841-857.

Fernández Pérez ER, Olson AL and Frankel SK (2011). Eosinophilic lung diseases. Med. Clin. North Am. 95: 1163-1187. Kalogeropoulos AS, Tsiodras S, Loverdos D, Fanourgiakis P, et al. (2011). Eosinophilic pneumonia associated with daptomycin: a case report and a review of the literature. J. Med. Case Rep. 5: 13.

Katz U and Shoenfeld Y (2008). Pulmonary eosinophilia. Clin. Rev. Allergy Immunol. 34: 367-371.

Martins RR, Marchiori E, Viana SL, Grillo Junior LS, et al. (2008). Chronic eosinophilic pneumonia secondary to longterm use of nitrofurantoin: high-resolution computed tomography findings. J. Bras. Pneumol. 34: 181-184.

Mayo JR, Müller NL, Road J, Sisler J, et al. (1989). Chronic eosinophilic pneumonia: CT findings in six cases. AJR Am. J. Roentgenol. 153: 727-730.

Ray S, Kundu S, Goswami M and Maitra S (2012). Tropical pulmonary eosinophilia misdiagnosed as miliary tuberculosis: a case report and literature review. Parasitol. Int. 61: 381-384.

Samitas K, Rådinger M and Bossios A (2011). Current update on eosinophilic lung diseases and anti-IL-5 treatment. Recent Pat Antiinfect. Drug Discov. 6: 189-205.

Souza CA, Müller NL, Johkoh T and Akira M (2006). Drug-induced eosinophilic pneumonia: high-resolution CT findings in 14 patients. AJR Am. J. Roentgenol. 186: 368-373.

Vijayan VK (2007). Tropical pulmonary eosinophilia: pathogenesis, diagnosis and management. Curr. Opin. Pulm. Med. 13: 428-433. 